\title{
Mesoscopic decoherence in Aharonov-Bohm rings
}

\author{
A.E. Hansen, A. Kristensen, S. Pedersen, C.B. Sørensen, and P.E. Lindelof \\ The Niels Bohr Institute, University of Copenhagen, Universitetsparken 5, DK-2100 Copenhagen, Denmark
}

(October 24, 2018)

\begin{abstract}
We study electron decoherence by measuring the temperature dependence of Aharonov-Bohm (AB) oscillations in quasi-1D rings, etched in a high-mobility GaAs/GaAlAs heterostructure. The oscillation amplitude is influenced both by phase-breaking and by thermal averaging. Thermal averaging is important when the temperature approaches the energy scale, on which the AB oscillations shift their phase. For the phase-breaking, it is demonstrated that the damping of the oscillation amplitude is proportional to the length of the interfering paths. For temperatures $T$ from 0.3 to $4 \mathrm{~K}$ we find the phase coherence length $L_{\phi} \propto T^{-1}$, close to what has been reported for open quantum dots. This might indicate that the $T^{-1}$ decoherence rate is a general property of open and ballistic mesoscopic systems.
\end{abstract}

PACS numbers: 73.23-b, 73.63.Nm

The understanding of decoherence in quantum mechanical systems gives valuable insight into the cross-over from quantum to classical behavior. Quantum phenomena like weak localization, universal conductance fluctuations and the Aharonov-Bohm effect, that are observed in mesoscopic electronic systems, make these systems well suited for studying decoherence. The loss of electron phase coherence is interesting in its own right because it reveals information about the fundamental physics of the electron scattering mechanisms. Moreover, from the perspective of possible phase-coherent mesoscopic electronic devicest, knowledge of phase-breaking length and time scales is crucial.

At low temperatures, electron-electron scattering is usually the dominating source of phase-breaking. In disordered 1D and 2D conductors, the loss of phase coherence at low temperatures has been studied intensively, both theoretically and experimentallye. In clean elestron systems, the number of investigations are ferer $\mathrm{B}$. $\mathrm{\theta}$. In $2 \mathrm{D}$, experiments consistent with the expected electronelectron sf $^{2}$ attering time $\tau_{\phi} \sim\left(T^{2} \ln T\right)^{-1}$ has been carried out 3 . In open quantum dots (a op system), an unexpected $T^{-1}$ contribution was found 5 . In general, phase breaking mechanisms in ballistic, mesoscopic systems of dimensionality less than 2 , are presently not well understood.

Aharonov-Bohm (AB) rings are obvious systems for probing phase coherence. Here, the interference of two electron paths leads to conductance oscillations of period $h / e$ [or frequency $e / h$ in the magnetic flux enclosed by the paths. The oscillation amplitude is a direct measure of the interference strength, and it has been used to study decoherence in disordered system\$. For $\mathrm{AB}$
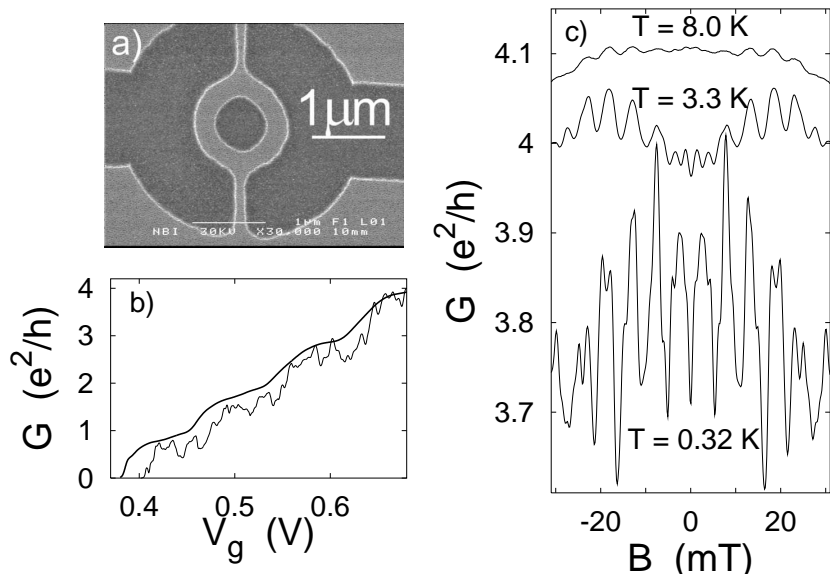

FIG. 1. a) SEM image of the ring before gate deposition. In the dark areas the donor layer is etched away. The quantum wires defining the arms of the ring are etched $280 \mathrm{~nm}$ wide, while the wires connecting the ring to the 2DEG reservoirs are $100 \mathrm{~nm}$ wide. b) Conductance $G$ vs. gate voltage $V_{g}$ at $T=5.8 \mathrm{~K}$ (thick line), and $0.32 \mathrm{~K}$ (thin line). c) Examples of $\mathrm{AB}$ oscillations measured at fixed gate voltage and different temperatures. Traces at high temperatures are offset for clarity.

rings with a 2DEG elastic mean free path longer than the circumference of the device (e.g.9 15 and references therein), systematic studies of phase-breaking have been scarce.

In this paper, we report measurements of the phase coherence length $L_{\phi}$ via the temperature dependence of $\mathrm{AB}$ conductance oscillations in quasi-1D rings, made by shallow etching in GaAs/GaAlAs heterostructures. Two mechanisms are important for the temperature dependence of the oscillation amplitude: phase-breaking, and thermal averaging. At finite temperature, the measured conductance is a weighted average over an energy interval of finite width, proportional to the temperature. We discuss, how thermal averaging influences the AB oscillation amplitude through the phase changes of the oscillations. In the experiment, we detect $\mathrm{AB}$ oscillations due to the interference of electrons that encircle the ring up to $n=6$ times. This is observed as peaks in the Fourier spectra of the magnetoconductance at multiples $n e / h$ of the fundamental $\mathrm{AB}$ frequency. Accounting for the effect of thermal averaging, we find that the damping of the amplitude due to phase-breaking depends linearly on $n$, showing directly the relaxation nature of the decoherence. We find the phase coherence length $L_{\phi} \propto T^{-1}$.

The $\mathrm{AB}$ rings are fabricated in a two-dimensional elec- 
tron gas (2DEG) situated $90 \mathrm{~nm}$ below the surface of a modulation doped GaAs/GaAlAs heterostructure. At liquid He temperatures, the unpatterned 2DEG density and mobility is $n=2.0 \times 10^{15} \mathrm{~m}^{-2}$ and $\mu=80 \mathrm{~m}^{2} / \mathrm{Vs}$, corresponding to an elastic mean free path $l_{e}=6 \mu \mathrm{m}$. The lateral confinement is obtained by a shallow wetetch, and the device is covered by a metal gate electrode. Details of the sample fabrication have been presented elsewhere12. The sample was cooled in a ${ }^{3} \mathrm{He}$ cryostat, and the conductance was measured in a two-terminal configuration. We used a conventional voltage biased lock-in technique, with an excitation voltage of $31.6 \mu \mathrm{V}$ oscillating at a frequency of $116.5 \mathrm{~Hz}$.

Two samples with identical designs have been investigated in detail. Here we present measurements on one of them. The main results are reproduced in the second sample. A Scanning Electron Microscope (SEM) image of the ring is shown in Fig. Ila. The ring has a circumference of $3 \mu \mathrm{m},<l_{e}$. The gate voltage dependence of the conductance is shown in Fig. 1 $1 \mathrm{~b}$. At $T=5.8 \mathrm{~K}$, the conductance increases in steps of height $\sim e^{2} / h$, due to the conductance quantization in the narrow exit and entrance wires2. The steps should not be taken as a sign of the population of transverse subbands in the ring itself, where the wires defining the arms are wider 6 . At $T=$ $0.3 \mathrm{~K}$, a UCF-type signal is superimposed on the steps, indicating a large degree of phase coherence in the ring at this temperature.

In Fig. Iic we show an example of the temperature evolution of the conductance in a perpendicular magnetic field $B$. The $\mathrm{AB}$ oscillation amplitude decreases with temperature. The period of the oscillations is $5.4 \mathrm{mT}$, in agreement with the period $h /\left(e \pi r^{2}\right)=5.5 \mathrm{mT}$ calculated from the average radius $r=490 \mathrm{~nm}$ of the ring.

To quantify the degree of coherence in the ring from the $\mathrm{AB}$ oscillations, we compute the Fast Fourier Transform (FFT) of the conductance $G(B)$, measured in the magnetic field interval as shown in Fig. 11c. A measure of the $\mathrm{AB}$ oscillation amplitude is obtained by integrating the $n e / h$ FFT peaks in the intervals as indicated in Fig. 2a. The amplitudes vary with gate voltage, as exemplified for the $e / h$ peak in Fig. 2b. It is sensitive to the phase difference $\Delta\left(k_{F} L\right)$ at zero magnetif field between electron paths in either arm of the device 17.12. The typical phase pickup in an arm is $k_{F} L \sim 200$, where $L=$ $1.5 \mu \mathrm{m}$ is half the circumference of the ring and $k_{F}$ is the Fermi wave number $\sim 1.5 \times 10^{8} \mathrm{~m}^{-1}$. A shift in the phase of the $\mathrm{AB} h / e$ oscillation, between the two values 0 and $\pi$ that are possible in a two-terminal measurement, requires $\Delta\left(k_{F} L\right)$ to change by $\pi$ 느. For a real device, $k_{F} L$ will not increase in exactly the same manner for the two arms, and hence the phase and the amplitude depends on the gate voltage, as we observe. The presence of several transverse subbands in the arms will provide an additional source of amplitude and phase variation.

The temperature dependence of the AB oscillation amplitude is shown in Fig. 20 for 10 different gate voltages. There is some scatter of the data points, around a well-
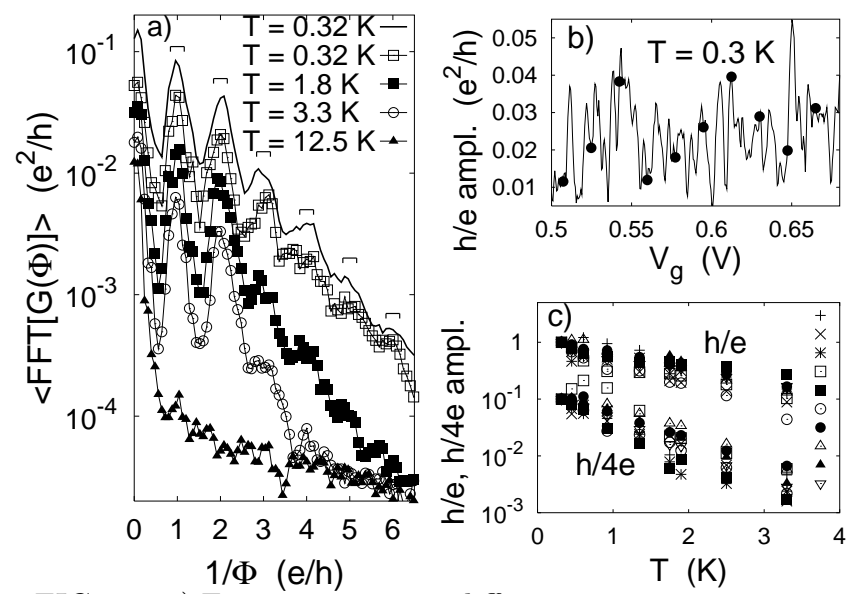

FIG. 2. a) Fourier spectra at different temperatures, given in the legend, as function of inverse magnetic flux, $\Phi=B \pi r^{2}$ through the ring. The spectra have been averaged for 10 different gate voltages. The spectrum marked with a thick line results from an average of 500 gate voltages and is offset vertically for clarity. The symbols mark the intervals in which the Fourier coefficients are integrated to calculate the oscillation strengths. b) Amplitude of $h / e$ oscillations as function of gate voltage. The filled circles mark the 10 gate voltages used in the further analysis. c) Amplitude of $h / e$ and $h / 4 e$ oscillations for 10 different gate voltages $V_{g}$, normalized to 1 and 0.1 at $T=0.3 \mathrm{~K}$, as function of temperature. The gate voltage increases from $V_{g}=0.51 \mathrm{~V}$ to $0.67 \mathrm{~V}$ in order of the symbols plotted to the right.

defined average. The temperature dependence of the oscillation amplitude does not depend strongly on gate voltage, in the gate voltage interval used here.

We take advantage of the weak gate voltage dependence, and perform an average of the Fourier spectra obtained at different gate voltages. In Fig. a a we show Fourier spectra averaged over the 10 gate voltages, for different temperatures. We also show an average Fourier spectrum computed from spectra obtained at 500 different gate voltages at $T=0.32 \mathrm{~K}$. Apart from the peak at the $e / h$ Aharonov-Bohm frequency, clear peaks appear at frequencies $2 e / h, 3 e / h, 4 e / h$, and smaller bumps are also visible at $5 e / h, 6 e / h$. Electrons can travel around the ring more than once, and a periodicity of $h / n e$ in flux of the conductance means that the interfering electron has enclosed the ring $n$ times. The probability for this type of event to happen will decrease with $n$, and so will also the amplitude of the $n e / h$ oscillation (as seen in Fig. 2a), with a rate that depends on the coupling between the ring and the 2DEG.

In Fig. 3a we show the temperature dependence of the amplitude of the $h / n e, n=1 \ldots 6$ oscillation periods, extracted from the average spectra as shown in Fig. 2a. For high temperatures and frequencies, the FFT amplitudes collapse onto a temperature-independent background spectrum. For temperatures between 0.3 and 4 $\mathrm{K}$, the amplitude drops exponentially with temperature for $n=1 \ldots 4$, but with different rates $b_{n}$ for different 

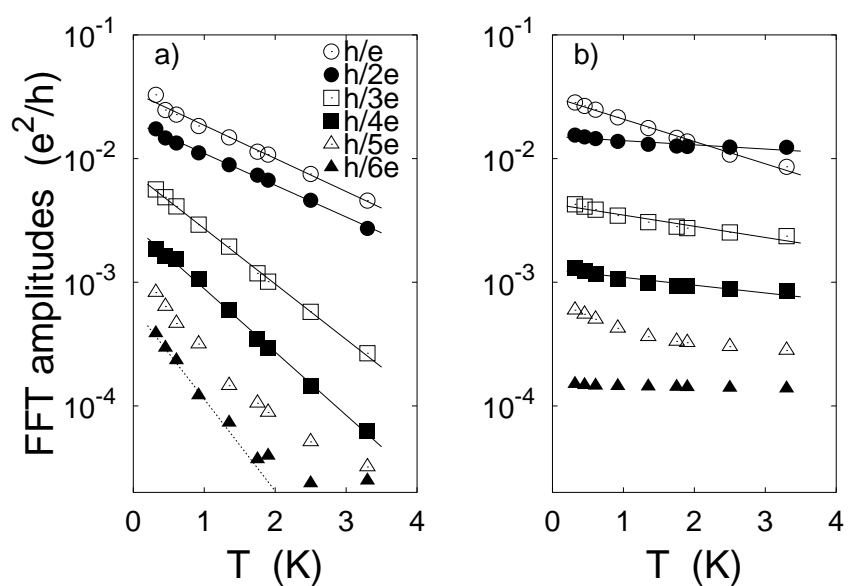

FIG. 3. a) Measured amplitudes of the $h / n e$ oscillations, $n=1 \ldots 6$, on a semi-log scale as function of temperature. Straight lines are fits with $a_{n} \cdot \exp \left(-b_{n} \cdot T\right)$, in the intervals that the lines cover. Dashed line shows the estimated decay rate of the $h / 6 e$ oscillation, $b_{6}=6 a$ (see Fig. (4). b) As in a), showing now amplitudes due to numerically calculated thermal averaging, as explained in the text. Straight lines are fits with $c_{n} \cdot \exp \left(-d_{n} \cdot T\right)$.

$n$. We assume that the amplitude of a $h / n e$ oscillation strength is damped due to phase-breaking in the following manner: 19

$$
\mathrm{h} / \text { ne amplitude } \propto e^{-n L / L_{\phi}(T)},
$$

where $L_{\phi}$ is the characteristic length over which correlations in the electron phase are lost. Eq. (Ii) implies that all $h / n e$ amplitudes should have the same functional dependence on $T$, as is observed. The measured exponential dependence means that $L_{\phi} \sim T^{-1}$. Furthermore, the damping rates $b_{n}$ (the slope of the lines in Fig. 3a) should increase linearly with $n$. This is not exactly the case, even though the rates do increase with $n$.

This apparent inconsistency between the data and the expected behavior due to phase-breaking, Eq. (11), can be understood when accounting for the effect of thermal averaging on the amplitude of the oscillations. At finite temperature, transport can take place in an energy window around the Fermi energy. In the Landauer-Büttiker formalism, the zero temperature conductance $G$ is then convoluted with the derivative of the Fermi function $f$,

$$
G\left(E_{F}, T, B\right)=\int d E G(E, 0, B)\left(-\frac{\partial f\left(E, E_{F}, T\right)}{\partial E}\right)
$$

Considering thermal averaging only, the amplitude of the $\mathrm{AB}$ oscillations will on average decrease with temperature, only if the oscillations at low temperature change phase within the available energy window, $\sim 3.5 k_{B} T$ wide. Phase-shifts of the $\mathrm{AB}$ oscillations, can occur if the geometrical phase difference, $\Delta\left(k_{F} L\right)$, between the two interfering paths changes as function of the Fermi energy, as has been motivated above. Therefore, the relevant temperature scale on which thermal averaging becomes efficient, is given by the Fermi energy change required to shift the phase of the $\mathrm{AB}$ oscillations, rather than for instance the energy level spacing of ring eigenstates.

We use Eq. (2) to estimate the effect of thermal averaging in our experiment. For $G\left(E_{F}, 0, B\right)$ we use a data set $G\left(V_{g}, T=0.32 \mathrm{~K}, B\right)$, where $V_{g}$ is changed in steps of $0.6 \mathrm{mV}$, small enough to resolve all the changes in the $\mathrm{AB}$ oscillations. The relation of gate voltage $V_{g}$ to Fermi energy $E_{F}$ is calibrated by following the spiky features seen on the low-temperature conductance curve in Fig. 11 b for finite bias voltages, $V_{s d}$. They will for small biases move linearly in the $\left(V_{g}, V_{s d}\right)$ - plane with a slope $\delta V_{s d} / \delta V_{g}$, which is related to the Fermi energy change with gate voltage as $\delta E_{F} / \delta V_{g}=e / 2\left(\delta V_{s d} / \delta V_{g}\right)$. The extracted $\delta E_{F} / \delta V_{g}$ is close to a simple capacitor estimate. From the simulated data sets $G\left(V_{g}, T>0.32 \mathrm{~K}, B\right)$, the average $\mathrm{AB}$ oscillation amplitude is extracted in the same manner as for the measured data. The result is shown in Fig. $3 \mathrm{~b}$. The calculated oscillation amplitudes do decrease with temperature, but much slower than the measured amplitudes. We conclude that thermal averaging alone can not account for the measured data. Furthermore, there is a clear tendency that the calculated $h / n e$ amplitudes decay faster for $n$ odd than even. For $n$ even, the magnetoconductance oscillations result partly from interference between time-reversed paths, the same paths that ultimately give rise to Altshuler-Aronov-Spivak oscillations in disordered systems 18 . The geometrical phase difference of these paths is zero, which means that the resulting part of the magneto-oscillations does not change phase. Consequently they are insensitive to thermal averaging. This explains why the even frequencies in Fig. $3 \mathrm{~b}$ have a slower temperature dependence than the odd frequencies. The temperature dependence can be approximated by exponentials, lines in Fig. $3 \mathrm{~b}$. For diffusive systems $\mathrm{b}$, thermal averaging is not important for temperatures below $E_{c} / k_{B}, \sim 15-20 \mathrm{~K}$ for our system, larger than the measurement temperatures. Here $E_{c}=\hbar \pi^{2} D /(2 L)^{2}$ is the standard expression for the correlation energy, and the diffusion coefficient $D=v_{F}^{2} \tau$ in 1D. But numerical calculations on a ballistic ring have given an enential temperature dependence for low temperatures 20 .

We can now return to the experimental results in Fig. 3a. As shown, the measured $h / e(h / 3 e)$ decay rate has a larger contribution from thermal averaging than the $h / 2 e$ $(h / 4 e)$ decay rate. We see that the part of the damping that thermal averaging can not account for, approaches the scaling with $n$ as foreseen in Eq. (1). To demonstrate this, we show in Fig. Ga the measured $h / n e$ decay rates vs. $n$, and in $4 \mathrm{~b}$ the estimated decay rates due to thermal averaging. The measured rates $b_{n}$ are not directly proportional to $n$. But the $h / 2 e, h / 4 e$ decay rates, which are only little influenced by thermal broadening (since $\left.b_{2} / d_{2}, b_{4} / d_{4} \sim 8\right)$, do obey the scaling (straight line). Even the $h / 6 e$ decay rate extrapolated from the scaling agrees with the data (dashed line in Fig. 3a). From the data in Fig. Ha-b it seems plausible, that with a proper deconvolution of the thermal averaging from the phase- 

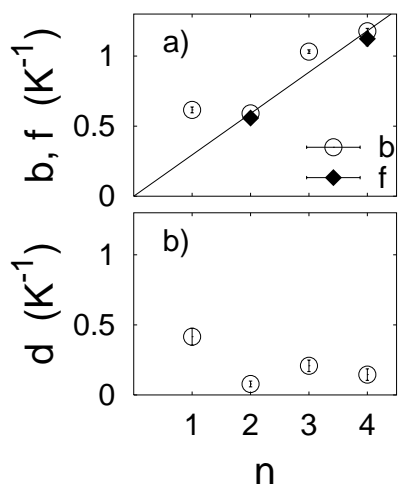
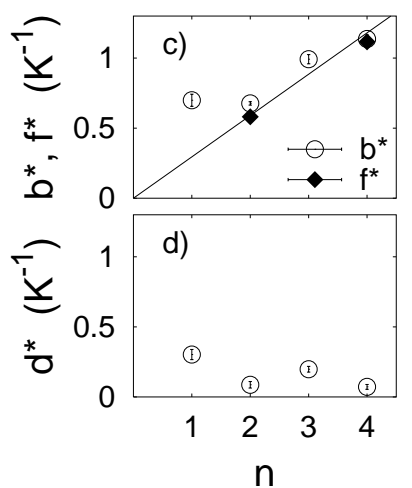

FIG. 4. a) Circles: exponents $b_{n}$ from the measured data Fig. Ba vs. $n$. Error bars on the symbols take into account the standard deviations on the fits. Straight line: fit with $b_{n}=\alpha \cdot n, \alpha=0.3 \mathrm{~K}^{-1}$ to the $n=2$ and $n=4$ points. Diamonds: exponents $f_{n}$ obtained from the temperature dependence of averaged AB oscillations, as described in the text. b) Exponents $d_{n}$ from the simulation Fig. $3 \mathrm{~b}$ vs. $n$. Error bars on the symbols take into account the standard deviations on the fits and the Fermi energy calibration. c) and d) as in a) and $b$ ), for another sample.

breaking, also the $h / e, h / 3 e$ decay rates will obey the scaling. The inclusion of the $n e / h$ frequencies, $n>1$, as shown in Fig. 1, gives a consistency check that the temperature damping of the $\mathrm{AB}$ oscillations results from a phase-breaking process, in addition to the effect of thermal averaging, and this supports the assumption Eq. (1). This has to our knowledge not been done before. As a further cross-check of the analysis we have also considered the amplitude of averaged, measured $\mathrm{AB}$ oscillations (as opposed to the average Fourier spectra, that has been used above). On performing the average, the amplitude of $h / n e$ oscillations for $n$ odd is strongly reduced. The temperature dependence of the amplitude of the ensemble averaged $h / 2 e, h / 4 e$ oscillations shows again an exponential decay. The exponents $f_{n}$ (diamonds in Fig. Aa) are only slightly smaller than the ones obtained from the ensemble averaged spectra, showing again that these oscillations are only little influenced by averaging. Finally, with the slope $0.3 \mathrm{~K}^{-1}$ of the line in Fig. Hawe deduce from Eq. (1) that $L_{\phi}(T=1 \mathrm{~K})=5 \pm 1 \mu \mathrm{m} 19$. Another way of expressing our result is that the damping of the interference amplitude $\sim \exp \left(-A L k_{B} T / \hbar v_{F}\right)$, where $A$ $\sim 0.4$. The same analysis performed on data from the second sample gives similar results (Fig. alc-d). Apart from these two rings, we have found interference amplitudes depending on temperature like $a \cdot \exp (-b \cdot T)$ in 5 other rings of different designs and sizes.

In summary, we have measured the temperature dependence of phase-breaking in quasi-1D AB ring structures. We have detected oscillation amplitudes resulting from the interference of electron paths encircling the ring more than once. This has enabled us to verify a basic property of phase breaking: the damping of the amplitude scales with the length of the interfering paths. We have in the analysis included the effect of thermal averaging, which is determined by the phase-shifts of the $\mathrm{AB}$ oscillations and hence by the asymmetry changes of the ring. We find that the phase-breaking length is proportional to $T^{-1}$, close to what has been measured in open quantum dots 5 . Thus, the $T^{-1}$ dependence of phase-breaking might be a general characteristic of mesoscopic ballistic systems. A theoretical effort to address this question is needed.

Acknowledgments. The authors wish to thank $\mathrm{H}$. Smith and K. Flensberg for useful discussions. This work was supported by the Danish Technical Research Council (grant no. 9701490), the Danish Natural Science Research Council (grant no. 9903274), and by the EU (LTR Programme Q-SWITCH, grant no. 30960). The III-V materials used were made at the III-V Nanolab, operated jointly by the Microelectronics center of the Danish Technical University and the Niels Bohr Institute fAFG, University of Copenhagen.

${ }^{1}$ G. Burkard, D. Loss and D.P. DiVicenzo, Phys. Rev. B 59, 2070 (1999)

${ }^{2}$ See e.g. references in 6 , and 8

${ }^{3}$ S.Q. Murphy, J.P. Eisenstein, L.N. Pfeiffer, K.W. West, Phys. Rev. B 52, 14825 (1995)

${ }^{4}$ A. Yacoby, M. Heiblum, H. Shtrikman, V. Umansky, D. Mahalu, Semic. Sci. Technol. 9, 907 (1994)

${ }^{5}$ J.P. Bird, K. Ishibashi, D.K. Ferry, Y. Ochiai, Y. Aoyagi, T. Sugano, Phys. Rev. B 51, 18037 (1995); D. P. Pivin, Jr., A. Andresen, J.P. Bird, D.K. Ferry, Phys. Rev. Lett. 82, 4687 (1999); C. Prasad, D.K. Ferry, A. Shailos, M. Elhassan, J.P. Bird, L.-H. Lin, N. Aoki, Y. Ochiai, K. Ishibashi, Y. Aoyagi, Phys. Rev. B 62, 15356 (2000)

${ }^{6}$ A.G. Huibers, M. Switkes, C.M. Marcus, K. Campman, A.C. Gossard, Phys. Rev. Lett. 81, 200 (1998); A.G. Huibers, S.R. Patel, C.M. Marcus, P.W. Brouwer, C.I. Duruöz, J.S. Harris, Jr., Phys. Rev. Lett. 81, 1917 (1998)

${ }^{7}$ C. Hodges, H. Smith, J.W. Wilkins, Phys. Rev. B 4, 302 (1971); G.F. Giuliani, J.J. Quinn, Phys. Rev. B 26, 4421 (1982); H. Fukuyama, E. Abrahams, Phys. Rev. B 27, 5976 (1983)

${ }^{8}$ See e.g. S. Washburn, R.A. Webb, Rep. Prog. Phys. 55, 1311 (1992); C. Kurdak, A.M. Chang, A. Chin, T.Y. Chang, Phys. Rev. B 46, 6846 (1992)

${ }^{9}$ C.J.B. Ford, T.J. Thornton, R. Newbury, M. Pepper, H. Ahmed, D.C. Peacock, D.A. Ritchie, J.E.F. Frost, G.A.C. Jones, Appl. Phys. Lett. 54, 21 (1989); C.J.B. Ford, A.B. Fowler, J.M. Hong, C.M. Knoedler, S.E. Laux, J.J. Wainer, S. Washburn, Surf. Sci. 229, 307 (1990)

10 J. Liu, W.X. Gao, K. Ismail, K.Y. Lee, J.M. Hong, S. Washburn, Phys. Rev. B 50, 17383 (1994)

${ }^{11}$ G. Cernicchiaro, T. Martin, K. Hasselbach, D. Mailly, A. Benoit, Phys. Rev. Lett. 79, 273 (1997)

${ }^{12}$ S. Pedersen, A.E. Hansen, A. Kristensen, C.B. Sørensen, 
P.E. Lindelof, Phys. Rev. B 61, 5457 (2000); J. Low Temp. Phys. 118, 457 (2000); Mat. Sci. Eng. B 74, 234 (2000); A.E. Hansen, S. Pedersen, A. Kristensen, C.B. Sørensen, P.E. Lindelof, Phys. E 7, 776 (2000)

${ }^{13}$ W.G. van der Wiel, S. De Francesci, T. Fujisawa, J.M. Elzerman, S. Tarucha, L.P. Kouwenhoven, Science 289, 2105 (2000)

${ }^{14}$ Yang Yi, M. Heiblum, D. Sprinzak, D. Mahalu, Hadas Shtrikman, Science 290, 779 (2000)

${ }^{15}$ M. Casse, Z.D. Kvon, G.M. Gusev, E.B. Olshanetskii, L.V. Litvin, A.V. Plotnikov, D.K. Maude, J.C. Portal, Phys. Rev. B 62, 2624 (2000)

16 The energy spacing $\Delta E_{0,1}$ of the first transverse subband in the exit and entrance wires is $\sim 9 \mathrm{meV}$. For the arms of the ring, we estimate $\Delta E_{0,1}$ to $1-3 \mathrm{meV}$.

17 M. Büttiker, in SQUID'85 - Superconducting Quantum Interference Devices and their Applications, edited by H.D. Haklbohm and H. Lübbig (Walter de Gruyter, Berlin, New York 1985), page 529.

18 B.L. Altshuler, A.G. Aronov, B.Z. Spivak, D.Yu. Sharvin, Yu.V. Sharvin, Pis'ma Zh. Eksp. Teor. Fiz. 35, 476 (1982) [JETP Lett. 35, 588 (1982)]

19 It can be argued, that $L$ should be a circumference rather than the length of an arm. In that case, we underestimate $L_{\phi}$ by a factor of 2 .

${ }^{20}$ M. Shin, K.W. Park, S. Lee, E.-H. Lee, Phys. Rev. B 53, 1014 (1996) 